\title{
The Relativistic Geometry of the Complex Matter Space
}

\author{
Reza Ahangar \\ Department of Mathematics, Texas A\&M University, Kingsville, TX, USA \\ Email: reza.ahangar@tamuk.edu
}

How to cite this paper: Ahangar, R. (2017) The Relativistic Geometry of the Complex Matter Space. Journal of Applied Mathematics and Physics, 5, 422-438. https://doi.org/10.4236/jamp.2017.52037

Received: December 30, 2016 Accepted: February 14, 2017 Published: February 17, 2017

Copyright $\odot 2017$ by author and Scientific Research Publishing Inc. This work is licensed under the Creative Commons Attribution International License (CC BY 4.0).

http://creativecommons.org/licenses/by/4.0/ (c) (i) Open Access

\begin{abstract}
Duality behavior of photons in wave-particle property has posed challenges and opportunities to discover other frontiers of fundamental particles leading to the relativistic and quantum description of matter. The speed of particles faster than the speed of light could not be recognized, and matter was always described as a real number. A new fundamental view on matter as a complex value has been introduced by many authors who present a paradigm that is shifted from real or pure imaginary particles to Complex Matter Space. A new assumption will be imposed that matter has two intrinsic components: i) mass, and ii) charge. The mass will be measured by real number systems and charged by an imaginary unit. The relativistic concept of Complex Matter Space on energy and momentum is investigated and we can conclude that the new Complex Matter Space (CMS) theory will help get one step closer to a better understanding toward: 1) Un-Euclidean description of Minkowski Geometry in the context of the Complex Matter Space, 2) transformation from Euclidean to Minkowski space and its relativistic interpretation. Finally, geometrical foundations are essential to have a real picture of space, matter, and the universe.
\end{abstract}

\section{Keywords}

Relativistic Geometry, Mass and Charge, Complex Matter Space, Un-Euclidean, Minkowski’s Geometry

\section{Introduction}

Several thousand years of human experiences in geometry, which was structured by Pythagoras and Euclid, has undergone challenges during the past few centuries. Among all were the attempts to prove Euclid's fifth postulate independently from other postulates and distinguish all undefined terms like point, line, plane, from terms that can be redefined like distances and parallel lines. 
In seeking the truth behind the parallel postulate in $19^{\text {th }}$ century, some alternatives and geometric models to Euclid's parallel postulate were presented.

In 1898, one of the great mathematicians of all time, David Hilbert presented a rigorous axiomatic approach to Euclidean Geometry (see Hilbert, 1902 [1]). Many others like Riemann, Bolyai, and Lobachevski created Un-Euclidean Geometry.

Thus, Hyperbolic Geometry was born and many geometrical models were developed (see Milnor, 1996 [2]).

Many were skeptical of the validity of this bizarre geometry which was axiomatically consistent and agreed with the models developed. But all these theories were not experimented physically to be consistent with the natural universe.

Certainly, in a small velocity for a shorter distance, Euclidean Geometry is a simplest model for our physical universe. But it is not the proper model for the entire universe particularly when the velocity of the object is near or approaches to the speed of light.

Einstein's General Relativity predicted that gravity alters the velocity of light and this causes the light ray to bend. Or, it can be interpreted that light travels in a geodesic line. This is a revolutionary conclusion that the physical space with the existing mass and gravity is not Euclidean (see Taylor et al., 2004 [3], and Feynman, 1963 [4]).

NASA Experiment, May 4, 2011: Einstein was right again. There is a spacetime vortex around Earth, and its shape precisely matches the predictions of Einstein's theory of gravity. Researchers confirmed these points at a press conference today at NASA headquarters where they announced the long-awaited results of Gravity Probe B (GP-B). "The space-time around Earth appears to be distorted just as general relativity predicts", says Stanford University physicist Francis Everitt, principal investigator of the Gravity Probe B mission (NASA, $2011[5])$.

A shortest path in traveling between two points can be defined by an arclength or geodesic line. It may not be considered as straight lines because acceleration creates distortion. Due to high speed of the particle or a constant curvature there may two possibilities. The yard stick or rulers can get longer or shrink as they move away from the stationary point (Lavenda, 2009 [6]).

A massive object in space curves the space and bends the light, when a photon moves past a star; it's the star's gravity that causes it to deflect, regardless of whether we consider photon a particle or wave.

All evidence indicating the existence of dark matter and dark energy suggests that we may need to redefine matter. As a result we present the following two postulates:

Complex Matter Space (CMS) (see Ahangar, 2014 [7] [8] [9])

- Postulate ( () : mass and charge are two intrinsic components of matter and they can be measured and determined in a complex plane. The real part is equal to the mass and the imaginary part is the charge of the particle. That is $M=m+\mathrm{i} q$.

- Postulate (II): In a one dimensional space, position and velocity are assumed 
to be real numbers and the rate of change of the position of a particle can be assumed to be faster than the speed of light.

According to de Broglie's duality of particles used to describe matter-wave characteristic of photons (see Mayer, 2007 [10] and Gauthier, 2009 [11]). It is well known that the complex plane can help us to explain the duality of waves and explain a wave with two components in the complex plane with real and imaginary components:

$$
\psi(\boldsymbol{r}, t)=\psi_{\text {real }}+\mathrm{i} \psi_{\text {imag }}
$$

We will refer the value $|\psi|^{2}$ as the intensity of the wave function where the magnitude of the complex number is denoted by $|\psi|=\sqrt{\left|\psi_{\text {real }}\right|^{2}+\left|\psi_{\text {imag }}\right|^{2}}$ [3].

Traveling Beyond the Speed of Light:

According to the "Special Theory of Relativity", it is not allowed to cross the boundary of the speed of light $c$, in the real universe. But if we can pretend from this assumption that we exist in the imaginary world then it may be allowed to go faster than light, since our conjugate space will be the real world.

In section two we present an introduction to the geometric structure of Complex Matter Space. We will show its relation with Minkowski Geometry and pseudo-Euclidean space.

In section three, we use a bilinear transformation in hyperbolic geometry. In section four, we investigate the hyperbolic rotation which is equivalent to Lorentz transformation. In section five, we will show that the relativistic view of geometry is consistent with hyperbolic geometry. As a conclusion, the law of cosines is redeveloped in a hyperbolic triangle.

\section{Geometrical Structure of the CMS}

Assume $V$ is a vector space and $T$ represents a bilinear transformation from $V \times V$ into the $R$. Let us select vectors $u=\left\langle u^{0}, u^{1}, u^{2}, \cdots u^{n}\right\rangle$ and $v=\left\langle v^{0}, v^{1}, v^{2}, \cdots, v^{n}\right\rangle$ in the vector space $V$, where all of the first $(n+1)$ components are real

$$
u \text { and } v \in V
$$

define the transformation $T$ from $V \times V \rightarrow R$ by

$$
T(u, v)=\sum_{k=0}^{k=n} u^{k} v^{k}=u^{0} v^{0}+u^{1} v^{1}+u^{2} v^{2}+\cdots+u^{n} v^{n}
$$

In the following theorem, we will show that the transformation $T$ defined in (2) is a bilinear transformation.

Theorem (1): The operator $T: V \times V \rightarrow R$ defined by (2) is a bilinear operator meaning that for any real numbers $a$ and $b$

$$
T\left(a u_{1}+b u_{2}, v\right)=a T\left(u_{1}, v\right)+b T\left(u_{2}, v\right)
$$

Proof: Notice that the superscripts are not exponents, thus

$$
\begin{aligned}
T\left(a u_{1}+b u_{2}, v\right) & =\sum_{k=0}^{k=n}\left[a\left(u_{1}^{k}\right)+b\left(u_{2}^{k}\right)\right] v^{k} \\
& =a \sum_{k=0}^{k=n}\left(u_{1}^{k}\right) v^{k}+b \sum_{k=0}^{k=n}\left(u_{2}^{k}\right) v^{k} \\
& =a T\left(u_{1}, v\right)+b T\left(u_{2}, v\right)
\end{aligned}
$$


which proves that $T$ is a bilinear operator that can be used to define the $\mathrm{Mi}$ kowski norm.

Also notice that the operator $T$ mapping a vector $u$ to itself will produce a value in $R$ :

$$
T(u, u)=\sum_{k=1}^{k=n}\left(u^{k}\right)^{2}=\left(u^{0}\right)^{2}+\left(u^{1}\right)^{2}+\left(u^{2}\right)^{2}+\cdots+\left(u^{n}\right)^{2}
$$

If the vector space $V$ is selected from real valued vectors then

$$
\forall k \in N, u^{k} \in R,\left(u^{k}\right)^{2} \geq 0
$$

implies that the relation (4), is a positive definite inner product, that is for nonzero vector $\mathrm{u}$, the transformation $T(u, u)>0$.

But when the vectors are selected from the complex field then the relation (4) can be one of the following cases:

i) $T(u, u)>0$, the transformation $T$ is positive definite, when the vector $u$ is in the region which is called space-like.

ii) $T(u, u)<0$ the transformation $T$ is negative definite, when $u$ is in the time-like, otherwise

iii) $T(u, u)=0$ and the transformation is called indefinite, when the vector $u$ belongs in the light-like.

Many questions exist to justify the operator $T$ for objects in complex space as well as explanation for the properties of the transformation $T$. The most important one is when $T$ is positive or negative definite and the operator $T$ from $R^{n+1} \times R^{n+1}-->R$ satisfies the properties of a metric space (see Naber, 1992 $[12])$.

To answer this question we need the definition of Metric Space first.

Metric Space: $A$ set $X$, whose elements we shall call points, is said to be a Metric Space if with any two points $P$ and $Q$ of $X$ there is associated a real number $\mathrm{d}(P, Q)$, called distance from $P$ to $Q$ such that

a) $\mathrm{d}(P, Q)>0$ if $P \neq Q, \mathrm{~d}(P, P)=0$,

b) $\mathrm{d}(P, Q)=\mathrm{d}(Q, P)$, and c) $\mathrm{d}(P, Q) \leq \mathrm{d}(P, R)+\mathrm{d}(R, Q)$ for any $R$ in $X$

One can verify that the transformation $T$ defined in (2), (3), and (4) fails the postulate (a) of metric space, due to the case where $T(u, v)$ can be negative definite.

Thus as a conclusion $T$ is not a Metric Space.

II) Special Complex Geometrical Structure: The standard Euclidean inner product of two vectors in (2) and (3) can be defined by the following matrix form

$$
u \cdot v=u^{t} I v
$$

where, $I$ is the identity matrix, $\boldsymbol{u}^{t}=\left\langle u^{0}, u^{1}, u^{2}, \cdots u^{n}\right\rangle, \boldsymbol{v}^{t}=\left\langle v^{0}, v^{1}, v^{2}, \cdots, v^{n}\right\rangle$, and superscript $t$ represents the transpose of these vectors.

To develop Minkowski space in (2) for $k=0$, consider special coordinate for

$$
u^{0}=v^{0}=\mathrm{i} c t
$$

where the symbol $i$ is the imaginary number $i=\sqrt{-1}$. The value $c$ is assumed to 
be a constant real number representing the speed of light.

Thus the operator $T: V \times V \rightarrow R$ defined by the following

$$
T(u, v)=\sum_{k=1}^{k=n} u^{k} v^{k}+(\mathrm{i} c t)(\mathrm{i} c t)=\sum_{k=1}^{k=n} u^{k} v^{k}-\left(c^{2} t^{2}\right)
$$

is also a bilinear operator. The relation (7) for $n=3$ is a four dimensional Minkowski space for events $u$ and $v$ and the transformation can be described by

$$
T(u, v)=\sum_{k=1}^{k=3} u^{k} v^{k}+(\mathrm{i} c t)(\mathrm{i} c t)=u^{1} v^{1}+u^{2} v^{2}+u^{3} v^{3}-c^{2} t^{2}
$$

\section{Properties of the Bilinear Operator}

i) The transformation $T: V \times V \rightarrow R$ generates Minkowski inner product space and

$$
\|\boldsymbol{u}\|^{2}=T(u, u)=\sum_{k=1}^{k=n}\left(u^{k}\right)^{2}-(c t)^{2}
$$

Proof: The transformation is indefinite if it is neither positive nor negative definite.

Notice that the relations (2), (3), and (4) will demonstrate the four dimensional Minkowski space.

We can use the relation (8) to establish Minkowski Norm as follows:

$$
\|\boldsymbol{u}\|^{2}=T(u, u)=\sum_{k=1}^{k=3}\left(u^{k}\right)^{2}-(c t)^{2}=\left(u^{1}\right)^{2}+\left(u^{2}\right)^{2}+\left(u^{3}\right)^{2}-(c t)^{2}
$$

where the Euclidean norm in $3 D$ real event: $r^{2}=\left(u^{1}\right)^{2}+\left(u^{2}\right)^{2}+\left(u^{2}\right)^{2}$ can change (10) into the following form of

$$
T(u, u)=r^{2}-(c t)^{2}=\left\langle\mathrm{i} c t, u^{1}, u^{2}, u^{3}\right\rangle I_{4 \times 4}\left\langle\mathrm{i} c t, u^{1}, u^{2}, u^{3}\right\rangle
$$

where I represents the identity matrix, as the identity matrix used in (6)

$$
I_{4 \times 4}=\left[\begin{array}{cccc}
1 & 0 & 0 & 0 \\
0 & 1 & 0 & 0 \\
0 & 0 & 1 & 0 \\
0 & 0 & 0 & 1
\end{array}\right] .
$$

The Minkowski inner product by the transformation $T(u, u)$ in (11) applied for event vector $u$ may be positive definite, negative definite, or indefinite.

\section{Orthogonal Subspace:}

When the transformation $T(u, v)=0$, then two vectors $\mathrm{u}$ and $\mathrm{v}$ are said to be orthogonal.

Assume a subspace $S \subset V$, such that

$$
S^{\perp}=\{v \varepsilon V: T(v, w)=0 \text { for all } w \varepsilon S\}
$$

It can be concluded that the set $S^{\perp} \subset S$ as a subset of $S$ is also a subspace of $S$ ?

In the next step we will explore the consistency of a two dimensional analogy of Minkowski-Hyperbolic system with Complex Matter space.

Proposition (1): Let $M(x, y)$ be a point on a hyperbola

$$
x^{2}-y^{2}=1
$$


For positive values of $x$ there exists a unique value of $u$ such that

$$
x=\cosh (u) \text { and } y=\sinh (u)
$$

Proof: Let us take the transformation, $u=\sinh ^{-1}(y)$. The result can be demonstrated by the geometric property of the hyperbolic identity

$$
x^{2}-y^{2}=\cosh ^{2} u-\sinh ^{2}(u)=1 .
$$

Notice that the relation (13) for $(x, y)$ is a two dimensional example in a complex plane when the transformation $y=c t$ is described by

$$
x^{2}+(\mathrm{i} c t)^{2}=1 \Leftrightarrow x^{2}-\left(c^{2} t^{2}\right)=1 .
$$

Pseudo-Euclidean Space: In our introduction it has been verified that the CMS transformation is not a metric space. We will show that this is a special pseudo Euclidean Space.

We define a general form of pseudo-Euclidean space as a finite dimensional $\mathrm{n}$-space together with a quadratic form $q$. The quadratic form defined for any vector with a suitable choice of basis $\left(e_{1}, e_{2}, \cdots, e_{n}\right)$ can be applied to a vector $u$ giving (with $1 \leq k<n$ )

$$
u=\sum_{k=1}^{n} u^{k} e_{k}=u^{1} e_{1}+u^{2} e_{2}+\cdots+u^{n} e_{n}
$$

for a quadratic form

$$
q(u)=\left(u^{1}\right)^{2}+\left(u^{2}\right)^{2}+\cdots+\left(u^{k}\right)^{2}-\left[\left(u^{k+1}\right)^{2}+\cdots+\left(u^{n}\right)^{2}\right]
$$

is called the magnitude of the vector $u$. When $k=1 \cdots n$, then it is Euclidean Space. Notice that the superscripts $k$, for convenience, selected to be from 1 to $n$. This is slightly different from the transformation (2), (3), and (4). We will have the general Minkowski Space when $k=0$.

In a pseudo-Euclidean space, unlike in a Euclidean space, there exist vectors with negative magnitude.

$$
q(u)=\|u\|^{2}=\left(u^{1}\right)^{2}+\left(u^{2}\right)^{2}+\cdots+\left(u^{n}\right)^{2}-\left(u^{0}\right)^{2}
$$

for all positive integer $k$ where, $0<k \leq n$.

A very important pseudo-Euclidean space is Minkowski's Space which is the mathematical setting in which Albert Einstein's theory of special relativity is conveniently formulated. The following is another example of pseudo-Euclidean space using Minkowski space, $n=4$ and $k=3$ so that

$$
q(u)=u_{1}^{2}+u_{2}^{2}+u_{3}^{2}+u_{4}^{2}
$$

where assuming $\|u\|=\sqrt{q(u)}$ and $u_{4}=\mathrm{i} c t$ the above relation will be

$$
\|u\|=\sqrt{u_{1}^{2}+u_{2}^{2}+u_{3}^{2}+(\mathrm{i} c t)^{2}}=\sqrt{u_{1}^{2}+u_{2}^{2}+u_{3}^{2}-(c t)^{2}}
$$

The geometry associated with this pseudo-metric was investigated by Poincare and the rotation group is called Lorentz group.

This magnitude is used in Lorentzian manifold which represents the flat space-time in $R^{4}$ uses the coordinates $(x, y, z, t)$ and the metric 


$$
\begin{aligned}
& \mathrm{d} s^{2}=\left[\begin{array}{llll}
\mathrm{i} c \mathrm{~d} t & \mathrm{~d} x & \mathrm{~d} x & \mathrm{~d} y
\end{array}\right]\left[\begin{array}{cccc}
1 & 0 & 0 & 0 \\
0 & 1 & 0 & 0 \\
0 & 0 & 1 & 0 \\
0 & 0 & 0 & 1
\end{array}\right]\left[\begin{array}{c}
\mathrm{i} \mathrm{d} t \\
\mathrm{~d} x \\
\mathrm{~d} y \\
\mathrm{~d} z
\end{array}\right] \\
& \mathrm{d} s^{2}=\mathrm{d} x^{\mu} 1_{4 \times 4} \mathrm{~d} x_{v} \\
& \mathrm{~d} s^{2}=(\mathrm{i} c \mathrm{~d} t)^{2}+\mathrm{d} x^{2}+\mathrm{d} y^{2}+\mathrm{d} z^{2}
\end{aligned}
$$

\section{From Euclidean Rotation to Lorentz Transformation}

When we replace $x^{2}$ symbolically for the sum of the squares of all real components and $u^{0}=c t$ in the relation (18) we will obtain the quadratic form

$$
q(u)=x^{2}-(c t)^{2}
$$

Definition of Lorentz transformation Matrix: We also use the relations (14) to define the following transformation:

$$
T(\boldsymbol{u})=\left[\begin{array}{cc}
\cosh (u) & \sinh (u) \\
\sinh (u) & \cosh (u)
\end{array}\right]
$$

where $x=\cosh (u)$ and $y=\sinh (u)$ imply that $b \tanh (u)=\frac{y}{x}$.

The transformation $T$ in (21) acting on an event vector $\left[\begin{array}{l}\zeta \\ l\end{array}\right]$ on a hyperbola $\zeta^{2}-\imath^{2}=k$ such that

$$
\left[\begin{array}{l}
t \\
z
\end{array}\right]=\left[\begin{array}{cc}
\cosh (u) & \sinh (u) \\
\sinh (u) & \cosh (u)
\end{array}\right]\left[\begin{array}{l}
\tau \\
\zeta
\end{array}\right]
$$

will be converted into a new hyperbola $z^{2}-t^{2}=k$ and preserves Minkowski's norm.

The concept of space and time has been evolved and became paradoxical from the time of Descarts to Minkowskiin twentieth century.

We are introducing the space-time coordinate $(x, y, z, t)$ such that it will transform into a new position point $\left(x^{\prime}, y^{\prime}, z^{\prime}, t^{\prime}\right)$.

The units on all components $x, y, z$ can be considered real numbers but we assume that the unit for time as an imaginary unit " $i c$ " such that $i=\sqrt{-1}$ and $c$ the speed of light $c$. Thus the component for $t$ can be demonstrated symbolically by

$$
\tau=\mathrm{i} c t
$$

To keep the concepts simple, assume y and $z$ stay fixed and the coordinate $(x \& \tau)$ under a rotation angle theta $=\theta$, then the new coordinate will be in the following form:

$$
\begin{aligned}
& x=x^{\prime} \cos (\theta)-\tau^{\prime} \sin (\theta) \\
& \tau=\tau^{\prime} \cos (\theta)+x^{\prime} \sin (\theta)
\end{aligned}
$$

or in the matrix form

$$
\left[\begin{array}{l}
x \\
\tau
\end{array}\right]=\left[\begin{array}{cc}
\cos (\theta) & -\sin (\theta) \\
\sin (\theta) & \cos (\theta)
\end{array}\right]\left[\begin{array}{l}
x^{\prime} \\
\tau^{\prime}
\end{array}\right]
$$


Let us call the initial reference frame $S=\{(\tau, x) \in C \times R\}$ and $S^{\prime}=\left\{\left(\tau^{\prime}, x^{\prime}\right) \in C \times R\right\}$ after rotation with angle theta. In this reference angle, the evolution on time axis is very interesting. The tau axis in the $S$ will be evolved into $(t a u)^{\prime}$ in the $S^{\prime}$ system. Any point on the tau axis will be transformed into a new point $(\mathrm{tau})^{\prime}$ in the equation when the angle theta is $90^{\circ}$.

In other words let us replace $x^{\prime}=0$ in the system (23) then

$$
\begin{gathered}
x=-\tau^{\prime} \sin (\theta) \\
\tau=\tau^{\prime} \cos (\theta)
\end{gathered}
$$

This is showing the transform of all of the points on the time axis. By dividing these two relations it will give us

$$
\tan (\theta)=-\frac{x}{\tau}
$$

Replace tau from the relation (4.1) we will obtain

$$
\tan (\theta)=-\frac{x}{\mathrm{i} c t}=\frac{\mathrm{i} v}{c}
$$

According to the relativity postulates, the time evolution is a function of the velocity. Thus we can find sine and cosine values of the angle theta using (26):

$$
\begin{gathered}
\cos (\theta)=\frac{1}{\sqrt{1+\tan ^{2}(\theta)}}=\frac{1}{\sqrt{1+\left(\frac{\mathrm{i} v}{c}\right)^{2}}}=\frac{1}{\sqrt{1-\left(\frac{v}{c}\right)^{2}}} \\
\sin (\theta)=\cos (\theta) \tan (\theta)=\frac{\frac{\mathrm{i} v}{c}}{\sqrt{1-\left(\frac{v}{c}\right)^{2}}}
\end{gathered}
$$

Substitute in the system (23)

$$
\left[\begin{array}{l}
x \\
\tau
\end{array}\right]=\gamma\left[\begin{array}{cc}
1 & -\frac{\mathrm{i} v}{c} \\
\frac{\mathrm{i} v}{c} & 1
\end{array}\right]\left[\begin{array}{l}
x^{\prime} \\
\tau^{\prime}
\end{array}\right]
$$

where gamma is equal to: $\gamma=\frac{1}{\sqrt{1-\left(\frac{v}{c}\right)^{2}}}$. The relation (27) is the Lorentz transformation. In addition to these sine and cosine functional transformations, one can develop the additively property of rotations and velocity:

$$
\tan (\theta+\phi)=\frac{\tan (\theta)+\tan (\phi)}{1-\tan (\theta) \cdot \tan (\phi)}=\mathrm{i} \frac{v+w}{1-v \cdot w}
$$

Minkowski investigated the Lorentz transformation as an Euclidean rotation. This can be achieved by finding $\left(x^{\prime}, \tau^{\prime}\right)$ from the system (23) and replacing (22) for tau.

$$
\left[\begin{array}{l}
x^{\prime} \\
\mathrm{i} c t^{\prime}
\end{array}\right]=\left[\begin{array}{cc}
\cos (\theta) & \mathrm{i} c t \cdot \sin (\theta) \\
-\sin (\theta) & \mathrm{i} c t \cdot \cos (\theta)
\end{array}\right]\left[\begin{array}{l}
x \\
\mathrm{i} c t
\end{array}\right]
$$


We also replace the angle theta by pure imaginary angle (iw) and using the following identities

$$
\cos (\theta)=\cos (\mathrm{i} w)=\cosh (w) \text { and } \sin (\theta)=\sin (\mathrm{i} w)=\mathrm{i} \sinh (w)
$$

Using these identities the hyperbolic version of the relation (28) will be

$$
\begin{aligned}
& \left\{\begin{array}{l}
c t^{\prime}=c t \cdot \cosh (w)-x \sinh (w) \\
x^{\prime}=-c t \cdot \sinh (w)+x \cosh (w)
\end{array}\right. \text { and the inverse } \\
& \qquad\left\{\begin{array}{l}
c t=c t^{\prime} \cdot \cosh (w)+x^{\prime} \cdot \sinh (w) \\
x=c t^{\prime} \cdot \sinh (w)+x^{\prime} \cdot \cosh (w)
\end{array}\right.
\end{aligned}
$$

These relations can be described by the following matrix form:

$$
\left[\begin{array}{c}
x^{\prime} \\
c t^{\prime}
\end{array}\right]=\left[\begin{array}{cc}
\cosh (w) & -\sinh (w) \\
-\sinh (w) & \cosh (w)
\end{array}\right]\left[\begin{array}{l}
x \\
c t
\end{array}\right]
$$

and

$$
\left[\begin{array}{c}
x \\
c t
\end{array}\right]=\left[\begin{array}{ll}
\cosh (w) & \sinh (w) \\
\sinh (w) & \cosh (w)
\end{array}\right]\left[\begin{array}{c}
x^{\prime} \\
c t^{\prime}
\end{array}\right]
$$

The hyperbolic systems in (30) explains the CMS with hyperbolic angle of rotation $w$ and rapidity

$$
\tanh (w)=\frac{v}{c}
$$

Comparing the two tangent relationships of (26) and (31) we can discover many interesting connections between two spaces i) Minkowski Space and ii) CMS.

One is in the real world and another in the imaginary space.

In the first we are dealing with Euclidean trigonometry and in the second with the hyperbolic trigonometry.

It can be concluded that

$$
\begin{aligned}
& \cosh (w)=\frac{1}{\sqrt{1-\left(\tanh (w)^{2}\right)}}=\frac{1}{\sqrt{1-\left(\frac{v}{c}\right)^{2}}}=\gamma \\
& \sinh (w)=\tanh (w) \cdot \cosh (w)=\frac{\frac{v}{c}}{\sqrt{1-\left(\frac{v}{c}\right)^{2}}}
\end{aligned}
$$

\section{-Geometry of Complex Matter in Complex Plane:}

Assume a complex point $z$ represents a particle $M=m+\mathrm{i} q$ such that

$$
|z|=m=\operatorname{Re}(M) \text { and } \arg (z)=\frac{q}{m}=\frac{\operatorname{Im}(z)}{|z|}=\arg (z)=\frac{\operatorname{Im}(z)}{\operatorname{Re}(z)}
$$

In this case we need to consider two different cases in which that can happen: When $|x|>|y|$ and $|x|<|y|$

Case (I): If $|x|>|y|$ then 


$$
r=|z|= \pm \sqrt{x^{2}-y^{2}}
$$

As a result: $\left(\frac{x}{r}\right)^{2}-\left(\frac{y}{r}\right)^{2}=\frac{x^{2}-y^{2}}{x^{2}-y^{2}}=1$

This statement describes the existence of an angle in Minkowskian Geometry such that

$$
\begin{gathered}
\cosh (\varnothing)=\frac{x}{r}=\frac{\operatorname{Re}(z)}{|z|}=\frac{m}{\sqrt{m^{2}+q^{2}}} \\
\sinh (\varnothing)=\frac{y}{r}=\frac{\operatorname{Im}(z)}{|z|}=\frac{q}{\sqrt{m^{2}+q^{2}}} \\
\tanh (\varnothing)=\frac{\sinh (\varnothing)}{\cosh (\varnothing)}=\frac{y}{x}=\frac{\operatorname{Im}(z)}{\operatorname{Re}(z)}=\frac{q}{m}
\end{gathered}
$$

case (II): If $|x|<|y|$ then

$$
\begin{gathered}
r=|z|= \pm \sqrt{y^{2}-x^{2}} \\
\left(\frac{y}{r}\right)^{2}-\left(\frac{x}{r}\right)^{2}=\frac{y^{2}-x^{2}}{y^{2}-x^{2}}=1
\end{gathered}
$$

Consequently, there exists a unique number phi such that:

$$
\begin{aligned}
& \sinh (\varnothing)=\frac{x}{r}=\frac{\operatorname{Re}(z)}{|z|}=\frac{m}{\sqrt{m^{2}+q^{2}}} \\
& \cosh (\varnothing)=\frac{y}{r}=\frac{\operatorname{Im}(z)}{|z|}=\frac{q}{\sqrt{m^{2}+q^{2}}} \\
& \tanh (\varnothing)=\frac{\sinh (\varnothing)}{\cosh (\varnothing)}=\frac{x}{y}=\frac{\operatorname{Re}(z)}{\operatorname{Im}(z)}
\end{aligned}
$$

As a result of this demonstration, we conclude that the complex number $z$ in CMS can be described symbolically by

$$
\left\{\begin{array}{l}
z=r(\cosh (\varnothing)+\mathrm{i} \sinh (\varnothing))=r * \operatorname{CISH}(\varnothing) \text { if }|x|>|y| \\
z=r(\sinh (\varnothing)+\mathrm{i} \cosh (\varnothing))=r^{*} \operatorname{SICH}(\varnothing) \text { if }|x|<|y|
\end{array}\right.
$$

\section{Relativistic and Minkowski Geometry in CMS}

The relations between Relativistic and Minkowski Geometry have been investigated extensively by many in the twentieth century. In this paper we would like to clarify the geometric concept and consistency of the Complex Matter Space with these two mathematical landmarks of the century.

By the relation (9) the Minkowski norm can be used to define an event vector

$$
\boldsymbol{p}=\left[p_{1}, p_{2}, \cdots, p_{n}\right]
$$

in the space-time $R^{n} \times C$ which is defined by

$$
E(\boldsymbol{p}, t)=\left[p_{1}, p_{2}, \cdots, p_{n}, \mathrm{i} t\right]
$$

where $R^{n}$ is real Euclidean space and $C$ is complex space, for $\mathrm{i}=\sqrt{-1}$. 
Definition of CMS inner-product: For two event vectors, $E_{1}=E(\boldsymbol{p})=\left(\boldsymbol{p}, t_{1}\right)$ and $E_{2}=E(\boldsymbol{q})=\left(\boldsymbol{q}, t_{2}\right)$ we define the CMS inner product by:

$$
E_{1} \cdot E_{2}=\sum_{k=1}^{n} p_{k} q_{k}+\left(\mathrm{it}_{1}\right)\left(\mathrm{i} t_{2}\right)
$$

As we defined in (18) and (19), the CNS pseudo-norm can be rearranged by

$$
\|E\|^{2}=\left(p^{1}\right)^{2}+\left(p^{2}\right)^{2}+\cdots+\left(p^{n}\right)^{2}-(t)^{2}=\|p\|^{2}-t^{2}
$$

This value can be positive, negative, or indefinite. Consequently, the vector event will be inside the time-like, light-like, or space-like.

Lemma 5.1: Assume two vector events $E_{1}$ and $E_{2}$, the inner product for CMS

$$
E_{1} \cdot E_{2}=\left(E_{1}\right)^{t} J E_{2}
$$

where $J$ is the $(n+1) \times(n+1)$ identity matrix.

Proof: By the definition:

$$
\begin{aligned}
& E_{1}=T\left(\boldsymbol{p}, t_{1}\right)=\left\langle\boldsymbol{p}, \mathrm{i} t_{1}\right\rangle \\
& E_{2}=E\left(\boldsymbol{q}, t_{2}\right)=\left\langle\boldsymbol{q}, \mathrm{i} t_{2}\right\rangle
\end{aligned}
$$

Using matrix multiplication, we can define

$$
\left.E_{1} \cdot E_{2}=\left(E_{1}\right)^{t} J E_{2}=\left[p_{1}, p_{2}, \cdots, p_{n}, \mathrm{it} 1\right]\left(\begin{array}{ccc}
1 & \cdots & 0 \\
\vdots & \ddots & \vdots \\
0 & \cdots & 1
\end{array}\right)\left[\begin{array}{c}
q_{1} \\
q_{2} \\
\vdots \\
q_{n} \\
\mathrm{it} t_{2}
\end{array}\right]=(\boldsymbol{p}, \mathrm{it})_{1}\right)^{t} J\left(\boldsymbol{q}, \mathrm{it} t_{2}\right)
$$

where the superscript $t$ represents the transpose of the matrix.

In the next step, we will use the development that used for the future events described in "The Geometry of Spacetime ..." by James Callahan (2000) [13].

We are targeting to apply for complex vector events which we need in the Complex Matter Space theory.

Event Transformation in CMS: Affine transformation includes translation and rotation that have been studied both in Euclidean and Minkowski geometries.

Let us denote the transformation of the, vector event $=\boldsymbol{r}$ by

$$
T_{\theta}(\boldsymbol{r})=A \boldsymbol{r}
$$

where $A$ is a matrix:

$$
A=\left[\begin{array}{ll}
\cosh (\theta) & \sinh (\theta) \\
\sinh (\theta) & \cosh (\theta)
\end{array}\right]
$$

With a little algebra one can verify the following inverse transform:

$$
A^{-1}=\left[\begin{array}{cc}
\cosh (\theta) & -\sinh (\theta) \\
-\sinh (\theta) & \cosh (\theta)
\end{array}\right]=\left(T_{\theta}(\boldsymbol{r})\right)^{-1}
$$

The angle $\theta$ of hyperbolic rotation in this transformation of the future vector $\boldsymbol{r}$ can be measured by hyperbolic radians. 
Lemma 5.1: The hyperbolic transformation $T$ has additive property on the rotation angle. That is

$$
T_{\theta}\left(T_{\alpha}\right) \equiv T_{\theta+\alpha}
$$

Proof: Assume a point $P$ is the initial position on a unit hyperbola representing the event $E=\boldsymbol{O P}=\left[\begin{array}{c}\cosh (\alpha) \\ \sinh (\alpha)\end{array}\right]$, such that the vector $P=(\cosh (\alpha), \sinh (\alpha))$

Thus using the definition,

$$
T_{\theta}(E)=T_{\theta}\left[\begin{array}{c}
\cosh (\alpha) \\
\sinh (\alpha)
\end{array}\right]=\left[\begin{array}{ll}
\cosh (\theta) & \sinh (\theta) \\
\sinh (\theta) & \cosh (\theta)
\end{array}\right]\left[\begin{array}{c}
\cosh (\alpha) \\
\sinh (\alpha)
\end{array}\right]
$$

By using algebra of multiplication of two matrices, we conclude that

$$
T_{\theta}(E)=\left[\begin{array}{c}
\cosh (\theta+\alpha) \\
\sinh (\theta+\alpha)
\end{array}\right]
$$

The new position is a new point $E^{\prime}$ on the hyperbola with the angle rotation alpha and the initial hyperbolic angle theta. That is $T_{\theta}(E)=E^{\prime}$.

Proposition 5.1: The Hyperbolic transformation in (39) preserves the CMS inner-product (40).

Proof: Assume two vector events $E_{1}=\left[P_{1}, t_{1}\right]^{t}$ and $E_{2}=\left[P_{2}, t_{2}\right]^{t}$. Using definition (37)

$$
\begin{aligned}
& T_{\theta}\left(E_{1}\right) \cdot T_{\theta}\left(E_{2}\right)=\left[T_{\theta}\left(E_{1}\right)\right]^{t} J T_{\theta}\left(E_{2}\right) \\
& =\left[E_{1}^{t} \cdot T_{\theta}^{t}\right] J T_{\theta}\left(E_{2}\right) \\
& =E_{1}^{t} \cdot\left[\begin{array}{cc}
\cosh (\theta) & \sinh (\theta) \\
\sinh (\theta) & \cosh (\theta)
\end{array}\right]\left[\begin{array}{cc}
1 & 0 \\
0 & -1
\end{array}\right]\left[\begin{array}{cc}
\cosh (\theta) & \sinh (\theta) \\
\sinh (\theta) & \cosh (\theta)
\end{array}\right] \cdot E_{2} \\
& =E_{1}^{t} J E_{2}=E_{1} \cdot E_{2}
\end{aligned}
$$

Proposition 5.2: Assume two vector events

$E_{1}^{t}=E\left(\boldsymbol{p}, t_{1}\right)=\left[p_{1}, p_{2}, \cdots, p_{n}, \mathrm{i} \cdot t_{1}\right]$, and $E_{2}^{t}=E\left(\boldsymbol{q}, t_{2}\right)=\left[q_{1}, q_{2}, \cdots, q_{n}, \mathrm{i} \cdot t_{2}\right]$ with a hyperbolic angle between the two event vectors $<E_{1} E_{2}=\beta$ then

$$
\boldsymbol{E}_{1} \cdot \boldsymbol{E}_{2}=\left\|\boldsymbol{E}_{1}\right\|\left\|\boldsymbol{E}_{2}\right\| \cosh (\beta)
$$

or equivalently; the angle between two hyperbolic events can be described by:

$$
\beta=\cosh ^{-1}\left(\frac{\boldsymbol{E}_{1} \cdot \boldsymbol{E}_{2}}{\left\|\boldsymbol{E}_{1}\right\|\left\|\boldsymbol{E}_{2}\right\|}\right)
$$

Proof: Let us assume the following two unit vectors for only two events:

$$
\boldsymbol{U}_{\iota}=\frac{\boldsymbol{E}_{\imath}}{\left\|\boldsymbol{E}_{\imath}\right\|} \quad \text { for } \quad i=1,2
$$

where the angle between the two unit vectors have the same direction as the event vectors

$$
\measuredangle \boldsymbol{U}_{1} \boldsymbol{U}_{2}=\measuredangle E_{1} E_{2}=\beta
$$

Select the transformation $T$ with initial hyperbolic angle theta such that 


$$
T_{\theta}\left(U_{1}\right)=\left[\begin{array}{l}
1 \\
0
\end{array}\right]
$$

to calculate the transformation of the second unit vector using the conclusion of (42):

$$
T_{\theta}\left(\boldsymbol{U}_{2}\right)=\left[\begin{array}{c}
\cosh (\theta+\beta) \\
\sinh (\theta+\beta)
\end{array}\right]=\left[\begin{array}{c}
\cosh (\beta) \\
\sinh (\beta)
\end{array}\right]
$$

Since the unit vector is in the direction of the horizontal axis when theta is zero we will find the second unit vector such that the hyperbolic rotation preserves the Minkowski inner product. That is

$$
\begin{gathered}
T_{\theta}\left(\boldsymbol{U}_{1}\right) \cdot T_{\theta}\left(\boldsymbol{U}_{2}\right)=\boldsymbol{U}_{1} \cdot \boldsymbol{U}_{2} \\
{\left[\begin{array}{l}
1 \\
0
\end{array}\right]^{t}\left[\begin{array}{c}
\cosh (\beta) \\
\sinh (\beta)
\end{array}\right]=\boldsymbol{U}_{1} \cdot \boldsymbol{U}_{2}} \\
\cosh (\beta)=\boldsymbol{U}_{1} \cdot \boldsymbol{U}_{2}=>\cosh (\beta)=\frac{\boldsymbol{E}_{1}}{\left\|\boldsymbol{E}_{1}\right\|} \cdot \frac{\boldsymbol{E}_{2}}{\left\|\boldsymbol{E}_{2}\right\|}
\end{gathered}
$$

Consequently we can have the final result:

$$
\boldsymbol{E}_{1} \cdot \boldsymbol{E}_{2}=\left\|\boldsymbol{E}_{1}\right\|\left\|\boldsymbol{E}_{2}\right\| \cosh (\beta)
$$

Proposition 5.3: Hyperbolic Triangular Inequality:

In every triangle $O E_{1} E_{2}$ when $\boldsymbol{E}_{1}$ and $\boldsymbol{E}_{2}$ are two event vectors with the angle between two vectors is $\measuredangle E_{1} E_{2}=\beta$ then

i)

$$
\left\|\boldsymbol{E}_{1}-\boldsymbol{E}_{2}\right\|^{2}=\left\|\boldsymbol{E}_{1}\right\|^{2}+\left\|\boldsymbol{E}_{2}\right\|^{2}-2\left\|\boldsymbol{E}_{1}\right\|\left\|\boldsymbol{E}_{2}\right\| \cosh (\beta)
$$

ii) The arc length connecting two points $E_{1}$ and $E_{2}$ :

$$
\left\|\boldsymbol{E}_{1} \boldsymbol{E}_{2}\right\|^{2}=\left\|\boldsymbol{E}_{1}\right\|^{2}+\left\|\boldsymbol{E}_{2}\right\|^{2}-2\left\|\boldsymbol{E}_{1}\right\|\left\|\boldsymbol{E}_{2}\right\| \cosh (\beta)
$$

Proof: (i) To prove the first relation (i), it would be helpful to see event vectors $\boldsymbol{E}_{1}-\boldsymbol{E}_{2}$ as a complex valued and their complex conjugates $\boldsymbol{E}_{1}-\boldsymbol{E}_{2}$ or $\left(\boldsymbol{E}_{1}-\boldsymbol{E}_{2}\right)^{*}$

$$
\begin{aligned}
\left\|\boldsymbol{E}_{1}-\boldsymbol{E}_{2}\right\|^{2} & =\left(\boldsymbol{E}_{1}-\boldsymbol{E}_{2}\right) \cdot\left(\boldsymbol{E}_{1}-\boldsymbol{E}_{2}\right)^{*} \\
& =\left(\boldsymbol{E}_{1}-\boldsymbol{E}_{2}\right) \cdot\left(\boldsymbol{E}_{1}^{*}-\boldsymbol{E}_{2}^{*}\right) \\
& =\boldsymbol{E}_{1} \boldsymbol{E}_{1}^{*}-\boldsymbol{E}_{1} \cdot \boldsymbol{E}_{2}^{*}-\boldsymbol{E}_{2} \cdot \boldsymbol{E}_{1}^{*}+\boldsymbol{E}_{2} \boldsymbol{E}_{2}^{*} \\
& =\left\|\boldsymbol{E}_{1}\right\|^{2}+\left\|\boldsymbol{E}_{2}\right\|^{2}-2 \boldsymbol{E}_{1} \boldsymbol{E}_{2}^{*}
\end{aligned}
$$

Substitute the conclusion in (45)

$$
\left\|\boldsymbol{E}_{1}\right\|^{2}+\left\|\boldsymbol{E}_{2}\right\|^{2}-2 \boldsymbol{E}_{1} \cdot \boldsymbol{E}_{2}=\left\|\boldsymbol{E}_{1}\right\|^{2}+\left\|\boldsymbol{E}_{2}\right\|^{2}-2\left\|\boldsymbol{E}_{1}\right\|\left\|\boldsymbol{E}_{2}\right\| \cosh (\beta) .
$$

ii) For the proof of the second part, consider $\left\|\boldsymbol{E}_{1} \boldsymbol{E}_{2}\right\|$ is the hyperbolic distance between two event vectors $E_{1}$ and $E_{2}$. The relation (47) is a direct conclusion of the

$$
\left\|\boldsymbol{E}_{1}-\boldsymbol{E}_{2}\right\|=\left\|\boldsymbol{E}_{1} \boldsymbol{E}_{2}\right\|
$$




\section{Concluding Remarks}

The Beltrami-Klein Model: It is helpful, first to try redefining the primitive terms like "point" "line", "lies on", and "between".

The Beltrami-Klein space is the set of all interior points of a circle which is called point in hyperbolic plane. Two points $A$ and $B$ inside a circle and a chord passing through these two points are imagined. The line is defined by an open chord joining these two points, which is called a hyperbolic line.

There exists an isomorphism between the Klein and Poincare models. That means there is a one-to-one correspondence can be set up between points and lines in one model and those in the others which preserve incidence, congruence, and betweenness.

By accepting the fifth postulate in Euclidean geometry, we can prove existence of a rectangle (quadrilateral with four right angles). Denying or having an alternative to the parallel postulate, particularly in hyperbolic geometry, one can prove that a triangle with angle sum equivalent to $2 \pi$ radians or quadrilateral with four right angles does not exist (see Figures 1-3).

Thus a quadrilateral with three right angles is called a Lambert Quadrilateral (see Figure 4).

Using the definition of hyperbolic tangent, it can be verified that the mapping $u-->\tanh (u)$ is a one-to-one mapping from the real line into the interval $(-1,1)$.

Notice that a free online software Geogebra used to draw the following graphs [14].

With a new advanced proposed postulate for a better unified approach in matter, energy, and momentum, we are challenged to connect the Complex Matter Theory to the two historical approaches of Relativity and Hyperbolic Geometry.

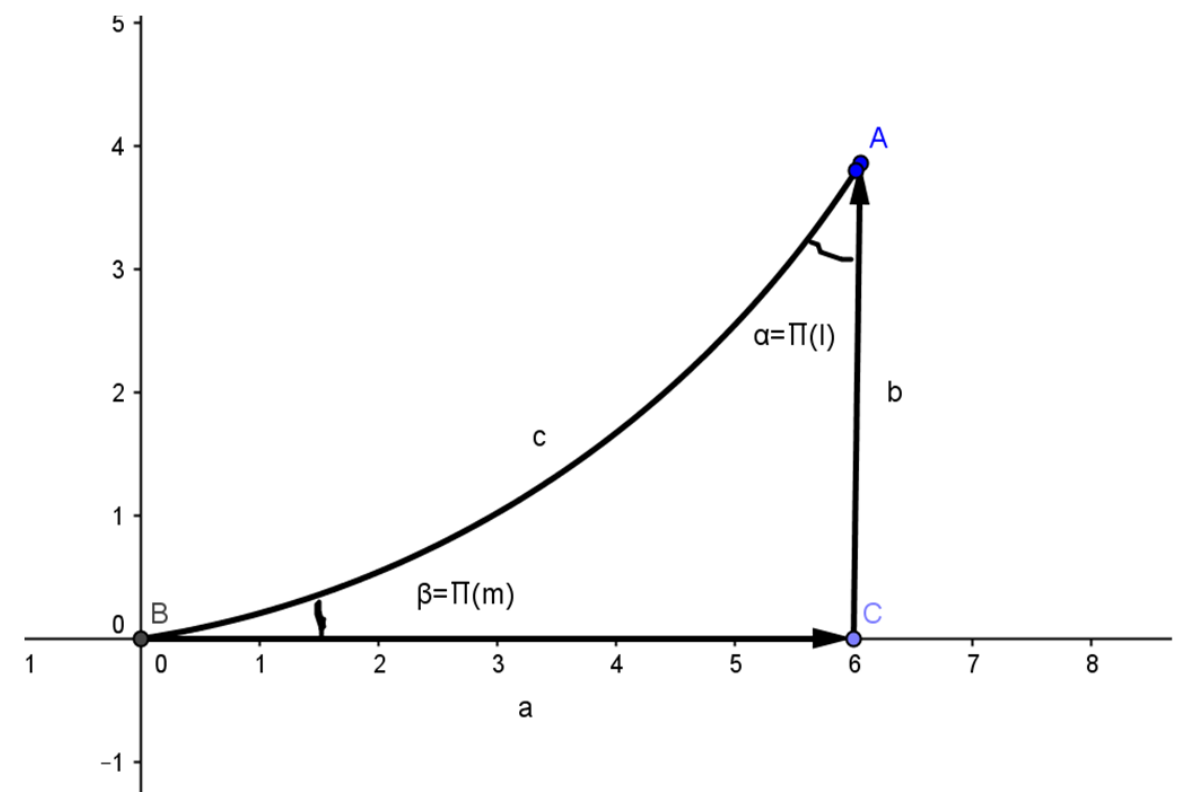

Figure 1. Hyperbolic right triangle with limiting angle of parallelism. 


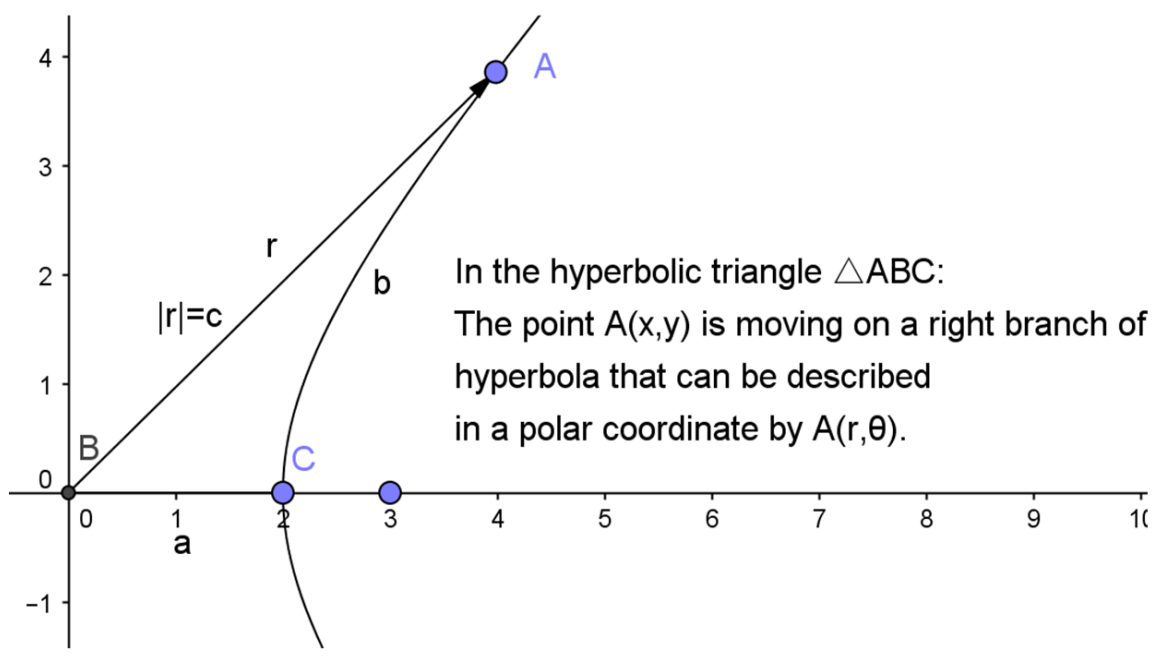

Figure 2. A point $A(x, y)$ is moving on a right branch of a unit hyperbola $x^{2}-y^{2}=1$, with a hyperbolic triangle $A B C$.

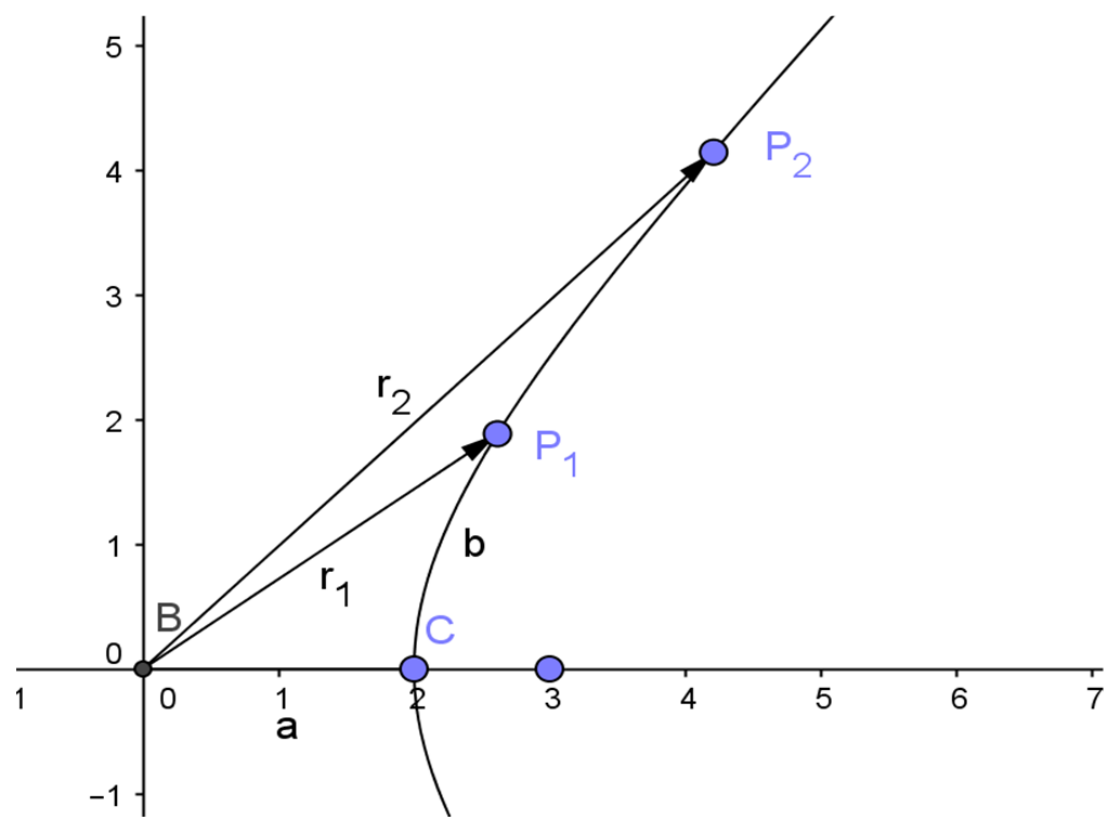

Figure 3. Two points $P_{1}$ and $P_{2}$ on the hyperbolic plane.

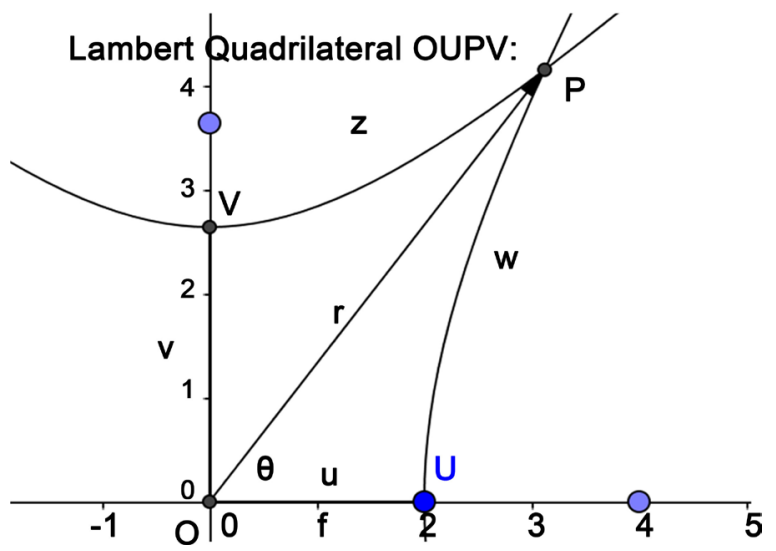

Figure 4. Lambert quadrilateral. 
In this work, we conclude that in the new complex matter approach, not only it is a new theory consistent with others, but it describes all shadows and unclear relations by a very beautiful method.

- Matter is redefined with two components of mass and charge which are the intrinsic part of the matter.

- Complex Matter Space Theory: all three space-time regions are space-like, time-like, and light-like. A particle like moving faster than the speed of light can be described by an imaginary mass and a particle-like with imaginary mass can travel faster than the speed of light.

- The geometry of the Complex Matter Space is consistent with the Lorentz transformation, Minkowski, and hyperbolic geometry.

- Given two vector events $\boldsymbol{E}_{1}$ and $\boldsymbol{E}_{2}$, the angle between two events can be described by a known formula $\cosh (\beta)=\frac{\boldsymbol{E}_{1} \cdot \boldsymbol{E}_{2}}{\left\|\boldsymbol{E}_{1}\right\|\left\|\boldsymbol{E}_{2}\right\|}$ in hyperbolic geometry.

- In every triangle $O E_{1} E_{2}$ when $\boldsymbol{E}_{1}$ and $\boldsymbol{E}_{2}$ are two event vectors with the angle that between two vectors is $\measuredangle E_{1} E_{2}=\beta$ then

$$
\left\|\boldsymbol{E}_{1}-\boldsymbol{E}_{2}\right\|^{2}=\left\|\boldsymbol{E}_{1}\right\|^{2}+\left\|\boldsymbol{E}_{2}\right\|^{2}-2\left\|\boldsymbol{E}_{1}\right\|\left\|\boldsymbol{E}_{2}\right\| \cosh (\beta)
$$

To compare these results with Euclidean space, one can see Greenberg (1980) [15] and Kay (2001) [16].

\section{References}

[1] Hilbert, D. (1902) The foundations of Geometry. The Open Court Publishing Company, Chicago, IL.

[2] Milnor, J. (1996) Hyperbolic Geometry: The First 150 Years. The Mathematics Heritage of Henry Poincare, Proceedings of Symposia in Pure Mathematics of the American Mathematical Society, 39, 25-40.

[3] Taylor, J., Zafiratos, C. and Dubson, M.A. (2004) Modern Physics for Scientists and Engineers. 2nd Edition, Pearson Prentice Hall, New Jersey.

[4] Richard, F. (1963) The Feynman Lectures on Physics. Addison Wesley, Reading, MA, Volume III, 1-6, 7-5.

[5] NASA (2011) Gravity Probe-B. https://science.nasa.gov/science-news/science-at-nasa/2011/04may_epic

[6] Lavenda, B.H. (2009) The Relativity of Hyperbolic Space. Universitadegli Studi, Camerino, Italy.

[7] Ahangar, R.R. (2014) Foundation of Complex Matter Space and Special Theory of Relativity, a Unifying Approach. Journal of Nuclear and Particle Physics, 4, 147153. http://article.sapub.org/10.5923.j.jnpp.20140405.03.html

[8] Ahangar, R.R. (2014) Quantum Complex Matter Space. International Journal of Theoretical and Mathematical Physics, 4, 159-163. http://article.sapub.org/10.5923.j.ijtmp.20140404.04.html

[9] Ahangar, R.R. (2014) Complex Matter Space and Relativistic Quantum Mechanics. Applied Mathematics, 5, No. 21. http://file.scirp.org/Html/11-7402454_52220.htm

[10] Mayer, A.F. (2007) Wave Energy in Quantum Mechanics. Journal of Physics, Conference Series, 70, 012013. https://doi.org/10.1088/1742-6596/70/1/012013 
[11] Gauthier, R. (2009) The Dirac Equation and the Superluminal Electron Model. http://www.superluminalquantum.org

[12] Naber, G.L. (1992) The Geometry of Minkowski Spacetime: An Introduction to the Mathematics of Special Theory of Relativity. Dover, New York.

[13] Callahan, J. (2000) The Geometry of Spacetime: An Introduction to Special and General Relativity. Springer, New York. https://doi.org/10.1007/978-1-4757-6736-0

[14] Geogebra Software. For Hyperbolic Geometry See. http://www.geogebra.org http://www.geogebra.org/material/simple/id/7005

[15] Greenberg, M.J. (1980) Euclidean and Non-Euclidean Geometries, Development and History. 2nd Edition, WH Freeman, New York.

[16] Kay, D.C. (2001) College Geometry a Discovery Approach. 2nd Edition, Addison Wesley Longman, Boston.

Submit or recommend next manuscript to SCIRP and we will provide best service for you:

Accepting pre-submission inquiries through Email, Facebook, LinkedIn, Twitter, etc. A wide selection of journals (inclusive of 9 subjects, more than 200 journals)

Providing 24-hour high-quality service

User-friendly online submission system

Fair and swift peer-review system

Efficient typesetting and proofreading procedure

Display of the result of downloads and visits, as well as the number of cited articles

Maximum dissemination of your research work

Submit your manuscript at: http://papersubmission.scirp.org/

Or contact jamp@scirp.org 